\title{
DIETZ ON GROUP-BASED REASONS
}

\author{
Magnus Jedenheim Edling
}

M ANY PEOPLE think not only that individuals have reasons to act, but that groups do too. ${ }^{1}$ Suppose that they are correct about this. Do the members of a group "inherit” the group's reason? Alexander Dietz has recently argued that they do so in some circumstances. ${ }^{2}$

Dietz considers two principles. The first one-which he calls the "Simple Principle"-claims that the members of a group always inherit the group's reason. The second one-which I call "Dietz's Principle," since it is the one Dietz advocates - claims that the members of a group inherit the group's reason when they cooperate. Although Dietz thinks that the Simple Principle is intuitively appealing, he argues that it has to be rejected because there is a powerful counterexample to it. In this article, I show that there is a powerful counterexample to Dietz's Principle as well.

I proceed as follows. In sections 1-2, I present the Simple Principle and Dietz's argument against this principle. In section 3, I introduce Dietz's Principle and show that it has the intuitively correct implications in the case that is a counterexample to the Simple Principle. In section 4, I turn to my case against Dietz's Principle. Finally, in section 5, I consider a natural revision of Dietz's Principle but conclude that it is unsatisfactory.

\section{THE SIMPLE PRINCIPLE}

The description I gave of the Simple Principle above was incomplete. It is not only concerned with reasons in favor of actions but also with reasons against actions. Here is the complete principle:

The Simple Principle: If a person is a member of a group such that the

1 For example, Dietz, "What We Together Ought to Do"; Jackson, "Group Morality"; and Tännsjö, “The Morality of Collective Actions.” Tännsjö does not think that people have reasons to act but is concerned with obligations to act.

2 Dietz, "What We Together Ought to Do." 
group has a reason (not) to perform a group action $\phi$, then that person has a reason (not) to do her part of $\phi .^{3}$

Some comments are in order. First, a group action is a combination of actions. If I perform action $A_{1}$ and you perform action $A_{2}$, you and I also perform the combination of actions $\left\langle A_{1}, A_{2}\right\rangle$. Furthermore, the combinations of actions a group of people can perform in some circumstances are a function of what individual actions the members of the group can perform in those circumstances. For example, if I can perform action $A_{1}$ and action $B_{1}$, and you can perform action $A_{2}$ and action $B_{2}$, you and I can together perform $\left\langle A_{1}, A_{2}\right\rangle,\left\langle A_{1}, B_{2}\right\rangle,\left\langle B_{1}, A_{2}\right\rangle$, $<B_{1}, B_{2}>$. There are several views on under what circumstances a combination of actions qualifies as a group action. For the sake of simplicity, I assume that all combinations of actions performed by at least two agents are group actions.

Second, Dietz thinks that the Simple Principle applies to several other moral reasons apart from reasons to make outcomes better; for example, reasons not to harm and reasons to benefit oneself. I am exclusively concerned with reasons to make outcomes better. Third, Dietz calls a reason that is inherited from a group's reason a "group-based" reason. Fourth, Dietz takes the Simple Principle to be an explanatory principle. For example, he takes the fact that a person is a member of a group that has a reason to perform a certain action to explain why that person has a reason to perform a certain action (i.e., his part of the group action in question).

\section{AN ARGUMENT AGAINST THE SIMPLE PRINCIPLE}

Dietz advances two arguments against the Simple Principle. ${ }^{4}$ For our purposes, it is sufficient that we consider what Dietz takes to be the most important one. ${ }^{5}$ This argument proceeds from the following case, which I call "Impending Disaster." A million lives are at risk and you and I face the following options (our actions are counterfactually independent):

3 Dietz, "What We Together Ought to Do," 969. Christopher Woodard has defended a view similar to the Simple Principle in the respect that it imposes no constraint to the effect that the parties should be willing to cooperate on the existence of group-based reasons (Reasons, Patterns, and Cooperation).

4 Dietz, "What We Together Ought to Do," 968-73.

5 Dietz suggests that there is a way around the other argument ("What We Together Ought to Do," 969-70). 
You

\begin{tabular}{|c|c|c|}
\hline & $A_{2}$ & $B_{2}$ \\
\hline$A_{1}$ & 100 saved & All die \\
\hline$B_{1}$ & All die & All saved \\
\hline
\end{tabular}

Let us first record the implications of the Simple Principle. It implies that I have one group-based reason against performing $B_{1}$ and one group-based reason against performing $A_{1}$ since you and I together have a reason against performing $<B_{1}, A_{2}>$ and a reason against performing $\left.<A_{1}, B_{2}\right\rangle$. (For our purposes, we may ignore your reasons.) This is so because each of these group actions would produce suboptimal outcomes if they were performed. According to Dietz, these two conflicting reasons "cancel each other out."

The Simple Principle also implies that I have a group-based reason in favor of performing $B_{1}$ since you and I together have a reason to perform group action $<B_{1}, B_{2}>$, which we have because $<B_{1}, B_{2}>$ would produce the optimal outcome in the circumstances if it were performed. Furthermore, it implies that I have a group-based reason against performing $A_{1}$ since you and I have a reason against performing group action $\left\langle A_{1}, A_{2}\right\rangle$, which we have since $\left\langle A_{1}, A_{2}\right\rangle$ would produce a suboptimal outcome if it were performed. These two reasons are not in conflict but rather point in the same direction. Although it will not be important here, it is plausible to assume that these two reasons do not add up.

In view of these remarks, it seems fair to say that the Simple Principle implies that I have one "effective" group-based reason in the case before us, namely, one in favor of performing $B_{1}$.

Now, suppose that you will actually perform $A_{2}$. In that case, apart from having an "effective" group-based reason in favor of performing $B_{1}$, I also have an ordinary reason to perform $A_{1}$ since otherwise one hundred more people will die. So, if you perform $A_{2}$, there is a conflict between my group-based reason to perform $B_{1}$ and my ordinary reason to perform $A_{1}$.

Since there is this conflict between these two reasons and since, intuitively, I ought to perform $A_{1}$ (since otherwise one hundred more people will die), proponents of the Simple Principle need a plausible view on the strength of groupbased reasons that generates this result. According to Dietz, it seems plausible that the strength of a person's group-based reason to perform an action is some proportion of the strength of the group's reason to perform the group action of which this action is a part.

6 Dietz, "What We Together Ought to Do," 979. 
According to this view, the strength of my group-based reason to perform $B_{1}$ is some proportion of the strength of the reason you and I together have to perform $<B_{1}, B_{2}>$. But what is the strength of this reason? According to Dietz, it "seems plausible that, if we are in a position to save lives, either together or individually, the strength of our reasons to do so will be proportional to the number of lives that would be saved." ${ }^{\text {C }}$ Consequently, the strength of our reason to perform $<B_{1}, B_{2}>$ is one million.

For the sake of argument, Dietz assumes that a person's group-based reason is "one thousandth as strong" as the group's reason. Given this assumption, it turns out that I ought to perform $B_{1}$ even if you perform $A_{2}$ since I then will have a group-based reason of strength one thousand to perform $B_{1}$ but only have an ordinary reason of strength one hundred to perform $A_{1}$. I have an ordinary reason of strength one hundred to perform $A_{1}$ if you perform $A_{2}$, since one hundred more people will be saved if I under those circumstances perform $A_{1}$. However, since intuitively I ought to perform $A_{1}$ if you perform $A_{2}$, the assumption that a person's group-based reason is "one thousandth as strong" as the group's reason must be wrong.

However, it would be no remedy to assume that a person's group-based reason might be a lot weaker than one thousandth as strong as the group's reason since a version of Impending Disaster may be constructed where many more than one million lives are at risk.

According to Dietz, the view that the strength of a person's group-based reason is some proportion of the strength of the group's reason should therefore be rejected. He briefly considers the view that there may be an upper bound on the strength of a person's group-based reason, but he rejects this view too. He argues that it is ad hoc, and that "setting the bound at any particular strength seems arbitrary."

Having considered this argument, Dietz concludes that the Simple Principle should be rejected.

\section{DIETZ'S PRINCIPLE}

As we just saw, the Simple Principle stumbles when someone fails to do her part of the group action that would produce the optimal outcome in the circumstances. To avoid this problem, Dietz adopts the following principle instead:

Dietz's Principle: If a person is a member of a group such that the group 
has a reason (not) to perform a group action $\phi$, and the group will perform $\phi$ if the person does her part of $\phi$, then that person has a reason (not) to do her part of $\phi .^{9}$

Dietz's Principle does not face the same difficulty as the Simple Principle faces. Consider again Impending Disaster. First, suppose that you perform $A_{2}$. If so, I have (as noted above) an ordinary reason to perform $A_{1}$ since otherwise one hundred more people will die. Of course, I have a group-based reason against performing $A_{1}$, according to Dietz's Principle, since you and I together have a reason against performing $\left\langle A_{1}, A_{2}>\right.$ and we will perform this action if I perform $A_{1}$ (since you perform $A_{2}$ ). However, I also have a group-based reason against performing $B_{1}$ since you and I together have a reason against performing $<B_{1}$, $A_{2}>$ and we will perform this action if I perform $B_{1}$ (since you perform $A_{2}$ ). And since my group-based reason against doing $B_{1}$ presumably is stronger than my group-based reason against doing $A_{1}$, the latter is "cancelled out."

Second, suppose that you perform $B_{2}$. In that case, I have an ordinary reason to perform $B_{1}$ since a million people will otherwise die. I also have a groupbased reason in favor of performing $B_{1}$, according to Dietz's Principle, since you and I together have a reason to perform $\left\langle B_{1}, B_{2}>\right.$ and we will perform that action if I perform $B_{1}$ (since you perform $B_{2}$ ). My group-based reason and my ordinary reason point in the same direction under these circumstances. I also have a group-based reason against performing $A_{1}$ since you and I together have a reason against performing $\left\langle A_{1}, B_{2}>\right.$ and we will perform this action if I perform $A_{1}$ (since you perform $B_{2}$ ). This reason is also in line with my ordinary reason to perform $B_{1}$.

\section{AN ARGUMENT AGAINST DIETZ'S PRINCIPLE}

My counterexample to Dietz's Principle is based on the following case, which I call the "Overdetermination Case": you and I, respectively, have two options. Each of us can either shoot the same person or refrain from doing so. If either of us shoots or if both of us shoot, the person dies, and if neither shoots, the person lives. Furthermore, our actions are counterfactually independent. Our situation is captured by the following:

9 Dietz, "What We Together Ought to Do," 977. My four remarks on the Simple Principle in section 1 also apply to Dietz's Principle.

10 This is so because the former is derived from a group action that you and I have a stronger reason against performing than the one from which the latter is derived. If there is an upper bound on the strength of a person's group-based reason, they may be equally strong. 


\begin{tabular}{r|l|l|}
\multicolumn{1}{c}{} & \multicolumn{1}{c}{ You shoot } & \multicolumn{1}{c}{ You do not shoot } \\
\cline { 2 - 3 } I shoot & The victim dies & The victim dies \\
\cline { 2 - 3 } I do not shoot & The victim dies & The victim lives \\
\cline { 2 - 3 } & &
\end{tabular}

Suppose that you will shoot. In that case, I have a group-based reason against abstaining from shooting, according to Dietz's Principle. First, you and I have a reason against performing $<\mathrm{I}$ do not shoot, you shoot $>$ since there is an alternative that has a better outcome. Second, we will perform $<$ I do not shoot, you shoot $>$ if I do not shoot since-I assume-you will shoot. However, intuitively, I do not have a reason against abstaining from shooting. ${ }^{11}$ This is my counterexample to Dietz's Principle.

Dietz might deny that it is counterintuitive that I have a reason against abstaining from shooting (if you shoot). However, that would not be a very plausible reply. According to Dietz's Principle, I have a group-based reason against abstaining from shooting (if you shoot) partly because you and I have a reason against performing $<$ I do not shoot, you shoot $>$. Moreover, you and I have a reason against performing this group action because there is an alternative whose outcome would be better. However, my abstaining from shooting does not contribute to the suboptimal outcome (i.e., that the victim dies) associated with $<\mathrm{I}$ do not shoot, you shoot>. In fact, it is necessary for the optimal outcome. In light of this, it would be implausible to insist that I have a reason against abstaining from shooting if you shoot.

Dietz might also reply that the fact that I have a group-based reason against abstaining from shooting does not determine what I ought to do and that it may therefore be ignored. This is because I also have a group-based reason against shooting (if you shoot), according to Dietz's Principle. You and I have a reason against performing $<$ I shoot, you shoot $>$ since there is an alternative that has a better outcome. Moreover, we will perform $<$ I shoot, you shoot $>$ if I shoot since-I assume-you will shoot. The upshot is (as above) that these two conflicting group-based reasons "cancel each other out." However, this reply is beside the point. It is still the case that, intuitively, I do not have a reason against abstaining from shooting.

Before I move on, I want to point out (although it might already be evident) that the case discussed in this section may be used against the Simple Principle

11 I think that this is true also if you and I have explicitly agreed to perform $<$ I do not shoot, you shoot> since I nevertheless do not contribute to the victim's death and since your action does not depend on mine. 
too. The Simple Principle also implies that I have a reason against abstaining from shooting even if you shoot since you and I have a reason against performing $<$ I do not shoot, you shoot $>$. Furthermore, I also want to note that Impending Disaster could also be used to raise a similar objection to the Simple Principle. As we saw, the Simple Principle implies that I have a group-based reason against performing $B_{1}$ in Impending Disaster (also if you perform $B_{2}$ ) since you and I have a reason against performing $\left.<B_{1}, A_{2}\right\rangle$. However, intuitively, I do not have a reason against performing $B_{1}$ (if you perform $B_{2}$ ).

\section{REVISING DIETZ'S PRINCIPLE}

Dietz might revise his principle. A suggestion that would not be promising is that Dietz should focus on reasons in favor of actions. Dietz might suggest that his principle should only be concerned with reasons in favor of actions and not also (as it is now) with reasons against actions. More precisely, he might suggest that we remove the two occurrences of "(not)" from his principle. I criticized Dietz's Principle because it implies, counterintuitively, that I have a group-based reason against abstaining from shooting (if you shoot), in the Overdetermination Case. This principle-i.e., the principle you get if you remove the two occurrences of "(not)" from Dietz's Principle-does not have this implication. As I noted, it is the fact that you and I have a reason against performing $<$ I do not shoot, you shoot $>$ and that we will perform this action if I abstain from shooting (if you shoot) that together with Dietz's Principle have this implication. However, as I said in the beginning of this paragraph, this would not be a promising suggestion. It would be an odd asymmetry if people were to inherit reasons in favor of actions but not also reasons against actions. If you inherit reasons in favor of actions it simply seems implausible that you do not also inherit reasons against actions.

However, there is a natural suggestion that might seem more promising. Dietz might suggest that we concentrate on those group actions the person herself can realize given the actual behavior of the other members. ${ }^{12}$ Suppose that I can perform action $A$ and action $B$, and that you can perform action $C$ and action $D$. Furthermore, suppose that you actually perform $C$. Under these circumstances, I can realize two group actions, namely, $\langle A, C\rangle$ and $\langle B, C\rangle$. Dietz might then suggest that I have a group-based reason in favor of performing $A$ if the outcome of $\langle A, C\rangle$ is better than the outcome of $\langle B, C\rangle$ and that I have a

12 I am grateful to a referee of this journal for the suggestion that Dietz might try to revise his view along these lines. 
group-based reason against performing $A$ if the outcome of $\langle A, C>$ is worse than the outcome of $\langle B, C\rangle$. In short, Dietz might propose the following principle:

The Revised Principle: A person has a reason (not) to do her part of a group action $\phi$ if that person can realize $\phi$ given the actual actions of the other members of the group, and the outcome of $\phi$ is (worse) better than the outcome of any other group action that the person can realize given the actual actions of the other members of the group.

Again, I criticized Dietz's Principle because it implies, counterintuitively, that I have a group-based reason against abstaining from shooting (if you shoot) in the Overdetermination Case. Just as the principle I briefly considered in the beginning of this section does not have this implication, the Revised Principle does not have it either. I can realize two group actions if you shoot: $<$ I do not shoot, you shoot $>$ and $<\mathrm{I}$ shoot, you shoot $>$. And the former does not have a worse outcome than the latter. Consequently, the Revised Principle does not imply that I have a group-based reason against abstaining from shooting if you shoot.

But there is another problem with the Revised Principle. As with the other principles discussed above, it is a principle about group-based reasons. It is supposed to tell us under what circumstances the members of a group inherit reasons to act from the group. A reasonable requirement for a person to inherit a reason to perform an action from the group is surely that this action is a part of a group action that the group has a reason to perform. A part can hardly inherit a feature from the whole that the whole lacks. According to the Revised Principle, however, a person may inherit a reason to perform a certain action although the group does not have a reason to perform the group action of which the action is a part.

Consider again Impending Disaster, for example. Suppose that you perform $A_{2}$. In that case, I have a group-based reason to perform $A_{1}$. Since you perform $A_{2}$, I can realize two group actions, namely, $\left\langle A_{1}, A_{2}\right\rangle$ and $\left\langle B_{1}, A_{2}\right\rangle$. And the former clearly has a better outcome than the latter. But from what group action is my group-based reason to perform $A_{1}$ supposed to be derived? There is one plausible candidate: $\left\langle A_{1}, A_{2}\right\rangle$. However, you and I do not have a reason to perform $<A_{1}, A_{2}>$ in the circumstances. The only group action you and I have a reason to perform is $\left\langle B_{1}, B_{2}>.^{13}\right.$ So, according to the Revised Principle, a person may in-

13 Although you and I may have a reason to perform $\left\langle A_{1}, A_{2}\right\rangle$ rather than $\left\langle B_{1}, A_{2}\right\rangle$, this is not relevant to our concerns here. We are concerned with the reasons we have to perform actions full stop. And in the circumstances, we only have a reason to perform $<B_{1}, B_{2}>$ full stop. 
herit a reason to perform an action although the group lacks a reason to perform the group action of which this action is a part.

To be sure, the Revised Principle implies that I have a group-based reason against performing $B_{1}$ if you perform $A_{2}$. Again, I can realize two group actions if you perform $A_{2}: \angle A_{1}, A_{2}>$ and $\angle B_{1}, A_{2}>$. And the latter has a worse outcome than the former. So, it may seem that I have a group-based reason to perform $A_{1}$ after all, since $A_{1}$ is the only alternative I have to $B_{1}$. Furthermore, importantly, my group-based reason against performing $B_{1}$ points in the same direction as the reason that you and I have against performing $\left\langle B_{1}, A_{2}>{ }^{14}\right.$ However, the explanation of why you and I have a reason against performing $\left\langle B_{1}, A_{2}>\right.$ has nothing to do with action $A_{1}$. We have this reason because $<B_{1}, B_{2}>$ has a better outcome.

Finally, note that Dietz's Principle does not suffer from the same shortcoming. It does not imply that I have a reason to perform $A_{1}$ if you perform $A_{2}$. As I said, according to Dietz's Principle, a person has a reason to do her part of a group action if the group of which the person is a member has a reason to perform the group action and this group will perform the group action if the person does her part. And you and I do not have a reason to perform $\left\langle A_{1}, A_{2}>{ }^{15}\right.$

Uppsala University magnus.jedenheim-edling@filosofi.uu.se

REFERENCES

Dietz, Alexander. "What We Together Ought to Do." Ethics 126, no. 4 (July 2016): 955-82.

Jackson, Frank. "Group Morality." Metaphysics and Morality: Essays in Honour of J.J.C. Smart, edited by Philip Pettit, Richard Sylvan, and Jean Norman. Oxford: Basil Blackwell, 1987.

14 As we just saw, my reason to perform $A_{1}$ does not point in the same direction as our reason to perform $<A_{1}, A_{2}>$. According to the Revised Principle, I have a reason to perform $A_{1}$, although you and I do not have a reason to perform $\left\langle A_{1}, A_{2}>\right.$.

15 I am grateful to participants in the Higher Seminar in Practical Philosophy at Uppsala University for very helpful comments. I give special thanks to Erik Carlson, Olle Risberg, Pekka Väyrynen, and a referee of this journal whose comments led to substantial improvements. Finally, I am particularly indebted to Jens Johansson for invaluable comments on most parts of this article. My research for this article was funded by a Göransson-Sandviken Research Scholarship awarded to me by Gästrike-Hälsinge Nation, Uppsala University. I am very grateful to them for their support. 
Tännsjö, Torbjörn. “The Morality of Collective Actions.” Philosophical Quarterly 39, no. 155 (April 1989): 221-28.

Woodard, Christopher. Reasons, Patterns, and Cooperation. New York: Routledge, 2008. 